\title{
Additions to the Flora of Garvin County, Oklahoma: Including a Complete Vascular Plant Checklist
}

\section{Priscilla H. C. Crawford and Phillip T. Crawford Oklahoma Biological Survey, University of Oklahoma, Norman, OK 73019}

A species list created from the Oklahoma Vascular Plants Database (OVPD) indicated that Garvin County, Oklahoma had been neglected by botanists. Our objective was to collect all vascular plant species encountered during three growing seasons to increase our botanical knowledge of the county. A total of 387 species were collected; 174 of these species had not been previously recorded for Garvin County in the OVPD. As a result, 14 families and 62 genera were added to the known flora of Garvin County. The majority of the species collected during this survey are assigned to five families of flowering plants: Poaceae (70 species), Asteraceae (63), Fabaceae (39), Cyperaceae (17), and Euphorbiaceae (16). Forty eight species (representing $13 \%$ of the taxa collected during this study) were not native to the United States; the families Poaceae and Fabaceae (with 18 and 10 species, respectively) exhibited the greatest number of introduced species collected in this survey. Five taxa on the Oklahoma Natural Heritage Inventory working list of rare Oklahoma plants were encountered. This collection effort increased the total number of vascular plant species recorded in the Oklahoma Vascular Plants Database for Garvin County to 628 species.

\section{INTRODUCTION}

During the last few decades there has been a decline in the number of plant specimens deposited in herbaria across the U.S. (Prather et al. 2004). This trend in plant collecting has been evident in Oklahoma as well (Hoagland et al. 2004). Certain areas, such as Black Mesa, Wichita Mountains, Arbuckle Mountains, LeFlore and McCurtain Counties, and the counties housing universities are well represented in the floristic collections of the state. Inconveniently located and less glamorous regions of Oklahoma have been ignored by the majority of botanists depositing plants in the state's herbaria, creating gaps in the known geographic distribution of taxa within the state. The objective of our research was to fill an apparent gap in floristic data for southcentral Oklahoma. G. W. Stevens made the first collections in Garvin County in 1913, and episodes of plant collecting in Garvin County have occurred in each decade since the 1960s (Figure 1). However, no single effort was made to collect all of the species found in a variety of habitats in Garvin County. Prior to our collection effort only 456 species were documented in the Oklahoma Vascular Plants Database (OVPD) for Garvin County, Oklahoma, compared to over 900 species recorded for neighboring McClain County (Hoagland et al. 2004). We believed that the relatively short OVPD species list for Garvin County was most likely due to a deficiency of plant collecting, rather than a lack of floristic diversity in the county.

\section{STUDY AREA}

The study area consisted of a privately owned farm and cattle ranch which covers 906.5 hectares (2240 acres) in the western portion of Garvin County, approximately 14.5 km (9 mi) south of Lindsay, Oklahoma (Fig. 2). Latitudinal extent ranges from $34.72^{\circ} \mathrm{N}$ to 
34.68 $\mathrm{N}$ and longitudinal extent from $97.56^{\circ} \mathrm{W}$ to $97.52^{\circ} \mathrm{W}$. The elevation ranges from 295-365 m (975-1195 ft). Rush Creek, which was straightened in this area in the early 1900 s, transects the northernmost portion of the study site, which has been continuously managed for livestock and crop production since 1904. The study site is in the Prairie Parkland Province of the Central Great Plains (Bailey 1995), although approximately 150 hectares (371 acres) has been cleared for cropland or "improved" pasture.

Physiographically, the study site is located in the Central Redbed Plains. The soils that dominate the site are loamy alluvium in the floodplain, loamy in the bottomland forest, and loamy clay soils in the upland (Kichler et al. 1982). The parent material is of Permian age and is dominated by shallow-marine, deltaic, and alluvial deposits of red sandstone and shale (Johnson et al. 1972). The study area is located within the Subtropical Humid (cf) climatic zone (Trewartha 1968). Mean annual precipitation is $93.5 \mathrm{~cm}$ (36.8 in) with May, June, September, and October being the wettest months $(21.6,10.7,10.2$, and $9.7 \mathrm{~cm}$ respectively). The mean annual temperature is $15.8^{\circ} \mathrm{C}\left(60.4^{\circ} \mathrm{F}\right)$; July and August are the hottest months, with average temperatures above $26.7^{\circ} \mathrm{C}\left(80^{\circ} \mathrm{F}\right)$. Temperatures exceed $32^{\circ} \mathrm{C}\left(90^{\circ} \mathrm{F}\right)$ approximately 70 days each year (Oklahoma Climatological Survey 2005).

\section{METHODS}

We conducted our survey during the growing seasons of 2001, 2003, and 2004. Although collections were made throughout the study site, areas for collection focus were selected with field reconnaissance and we returned to those specific areas at regular intervals during the growing season. A few collections were also made randomly throughout the county. The habitat type in which each plant was found was recorded, as was its relative abundance within the study site [see Palmer et al. (1995) for a detailed description of the abundance scale].
Specimens were identified using the following manuals: Waterfall (1969), Great Plains Flora Association (1986), Diggs et al. (1999), and Tyrl et al. (2002). Specimens from the Robert Bebb Herbarium at the University of Oklahoma (OKL) were consulted to confirm the identification of some collections. Nomenclature follows the National PLANTS Database (USDA, NRCS 2004). Voucher specimens for all species and, in certain cases, subspecific specimens were collected and deposited in the Robert Bebb Herbarium. Species were identified as native (originating in North America), introduced (originating outside of North America), or cultivated (species generally found only in cultivation; USDA, NRCS 2004). All non-native and agricultural species were collected from populations that had naturalized or were escaped from cultivation. Rarity status was determined using the Oklahoma Natural Heritage Inventory's list of rare plants of Oklahoma (Oklahoma Natural Heritage Inventory 2003). We compared our species list with the plant collection records for Garvin County found in the OVPD (Hoagland et al. 2004).

\section{RESULTS and DISCUSSION}

We collected 471 specimens representing 387 species of vascular plants; 174 of these species were not previously recorded in the OVPD for Garvin County (Appendix I). As a result, 14 families and 62 genera were added to the known flora of Garvin County. The greatest number of species collected during this study are assigned to the Poaceae (70 species), Asteraceae (63), Fabaceae (39), Cyperaceae (17), and Euphorbiaceae (16); these five families represented over $53 \%$ of the taxa collected at the study site. The remaining 83 families were each represented by 10 or fewer species. We combined our data with the floristic data found in the OVPD to produce a species list for Garvin County. There have been 628 species collected in Garvin County 
since 1913 (Table 1). The Asteraceae (98), Poaceae (89), and Fabaceae (63) are the three families with the largest number of species collected. These three families represent $40 \%$ of the species known to occur in the county.

We encountered no federally listed threatened or endangered species. However, we found several species tracked by the Oklahoma Natural Heritage Inventory: Eriogonum alatum (G5 S2S3); Mublenbergia bushii (G5 S1S2); Quercus sinuata var. breviloba (G5GT S?); Quercus stellata (G5 S?); and Rhynchospora nivea (G4 S2). Species are ranked according to their imperilment at both the global $(G)$ and state (S) levels; on the 5-1 scale, 5 indicates a species is secure and 1 indicates it is imperiled (Oklahoma Natural Heritage Inventory 2003).

Because older botanical work generally ignores introduced or "weedy" plants, we expected our collection to have a significantly higher proportion of these species than those of previous collectors. Prior to our study, only 46 species of introduced plants were represented in the OVPD for Garvin County, comprising 10\% of the taxa known to occur there. Our collection included 48 introduced species (including 26 species not previously recorded in the OVPD for Garvin County), representing $13 \%$ of the taxa collected during this study. Poaceae (18) and Fabaceae (10) were the families with the greatest number of introduced species.

We defined eight general habitat types found within the study area. Habitat type was recorded for each species (Appendix I). A brief description of each habitat type follows.

\section{Bottomland Forest (BF)}

Bottomland forest occurred in the floodplain of Rush Creek and along its principal tributaries. Hardwood trees such as Carya illinoinensis, Celtis laevigata, Fraxinus pennsylvanica, Quercus macrocarpa, Q. mueblenbergii, Q. velutina, and Ulmus americana dominated the canopy. The shrub layer consisted of many woody vines, including Passiflora spp., Parthenocissus quinquefolia, Smilax spp. and Vitis spp. Viola affinis, Chasmanthium latifolium, and Elymus canadensis were often found in the herbaceous layer of the bottomland forest.

\section{Upland Forest (UF)}

Upland forests differed from the bottomland forest by having a more open canopy and inhabiting coarser soils with less available moisture. Tree species dominating this forest were: Gleditsia triacanthos, Quercus marilandica, Q. stellata, and Sideroxylon lanuginosum. Common small trees and shrubs were: Cercis canadensis, Cocculus carolinus, Maclura pomifera, Morus rubra, Prunus mexicana, Sapindus saponaria var. drummondii, Symphoricarpos orbiculatus, and Viburnum rufidulum.

\section{Native Pasture (NP)}

Native Pasture occurred in upland areas that were originally open grasslands or areas of Cross Timbers that had been cleared of much of the woody vegetation. Native grasses dominated this habitat type: Andropogon gerardii, Bouteloua spp., Eragrostis spp., Schizachyrium scoparium, and Sorghastrum nutans. Common forb species of the native pasture were Ambrosia psilostachya, Amphiachyris dracunculoides, Desmanthus illinoensis, and Tetraneuris linearifolia. Some native prairie plants found were Asclepias tuberosa, Dalea enneandra, Gaillardia aestivalis, Liatris squarrosa, Ratibida columnifera, and Sabatia campestris. Small patches of the woody shrubs Prunus gracilis and Rhus glabra could be found within the native pasture, as well as two species of cacti (Escobaria missouriensis and Opuntia macrorbiza). Outcroppings of native sandstone hosting a different suite of species occurred within this habitat; many of these species (including Ceanothus americanus, Eriogonum alatum, Euphorbia longicruris, Oenothera macrocarpa ssp. okelahomensis, and Pellaea atropurpurea) were only observed in association with these rock outcrops (RO).

\section{Cultivated Pasture (CP)}

Cultivated pasture was typically found in areas previously inhabited by bottomland forest. These areas were cleared of woody 
vegetation and planted in row crops in the late 1800 s and early 1900s, and were planted with a monoculture of Cynodon dactylon (Bermuda grass) in the mid-1900s. Many "weedy" and introduced plants could be found within this habitat type. Other grasses encountered included Bromus spp., Cenchrus spinifex, and Setaria viridis. Typical forbs found in the cultivated pasture were Capsella bursa-pastoris, Galium aparine, Geranium carolinianum, and Lamium amplexicaule.

\section{Disturbed (D)}

Disturbed areas occurred throughout the study site and included roadsides, edges of cultivated fields, mowed lawns, areas experiencing heavy cattle usage (near feeding areas, stock tanks, or shade trees) and other areas exhibiting signs of physical disturbance. Disturbed areas share many species with the cultivated pasture habitat. Common forb species encountered were Ambrosia trifida, Cirsium undulatum, Conyza canadensis, Cucurbita foetidissima, Grindelia papposa, and Solanum elaeagnifolium. Aegilops cylindrica, Eragrostis cilianensis, Poa annua, and Sorghum balepense were grasses typical of disturbed areas, as were the escaped cultivated plants Secale cereale and Triticum aestivum.

\section{Riparian (R)}

The riparian zone was found along Rush Creek and its principal tributaries. Acer negundo, Celtis laevigata, Salix nigra, and Ulmus americana were the most abundant arborescent species in the riparian zone; Tamarix chinensis and Salix exigua were common along Rush Creek. Fruticose and herbaceous species abundant in the riparian zone included Amorpha fruticosa, Cephalanthus occidentalis, Erigeron philadelphicus, Eupatorium serotinum, Symphyotrichum oolentangiense, and Teucrium canadense.

\section{Seeps (S)}

Seeps occur sporadically within the native pasture, in areas where sloping rock strata carrying ground water from higher elevations intersect the soil surface. The flora of these seeps was conspicuously different from that of other wetland areas within the study site and that of the surrounding pastureland. This flora was dominated by graminoids, including several genera of sedges and rushes (Cyperus, Eleocharis, Fuirena, Juncus, Rhynchospora, Schoenoplectus, and Scirpus). A few grass species also occurred in this habitat (Andropogon glomeratus, Panicum anceps, and $P$. virgatum), as did several dicotyledonous forbs (Lobelia siphilitica, Marshallia caespitosa, and Valerianella amarella).

\section{Wetland and Aquatic (WA)}

Wetland and aquatic habitats were formed in and around farm ponds, beaver ponds on small drainages, springs, and in small depressions in native and cultivated pastures. Farm ponds and small depressions in pastures were typically bordered by a narrow fringe of herbaceous species common to frequently-flooded and disturbed habitats, and were subject to frequent trampling by cattle. The flora of these areas included Alopecurus carolinianus, Ammannia coccinea, Echinochloa crus-galli, Eclipta prostrata, Leucospora multifida, Mollugo verticillata, Phyla lanceolata, and Symphyotrichum subulatum. The few springs within the study site and several small impoundments created by beavers (Castor canadensis) on unnamed tributaries of Rush Creek were relatively undisturbed; these areas were flanked by woody vegetation similar to that of the above-described riparian zone. The beaver ponds were bordered by a narrow fringe of herbaceous vegetation, including Bidens frondosa, Equisetum byemale, E. laevigatum, Leersia oryzoides, L. virginica, Leptochloa fusca, Polygonum spp., and Ranunculus sceleratus. The springs were vegetated with the aquatic plants Potamogeton nodosus, Rorippa nasturtium-aquaticum, and Samolus valerandi.

\section{ACKNOWLEDGMENTS}

We thank Bruce Hoagland for verifying the identification of several plant specimens and making comments on earlier drafts of this manuscript. 
Table 1 Summary of Garvin County floristic collections since 1913

\begin{tabular}{lllllc}
\hline Taxonomic Group & Families & Genera & Native Spp. Exotic Spp. & Total Spp. \\
\hline Filicopsida & 4 & 5 & 6 & 0 & 6 \\
Equisteopsida & 1 & 1 & 3 & 0 & 3 \\
Pinopsida & 1 & 1 & 1 & 0 & 1 \\
Magnoliopsida & 79 & 273 & 413 & 41 & 454 \\
Liliopsida & 16 & 73 & 143 & 21 & 164 \\
TOTALS & 101 & 353 & 566 & 62 & 628 \\
\hline
\end{tabular}

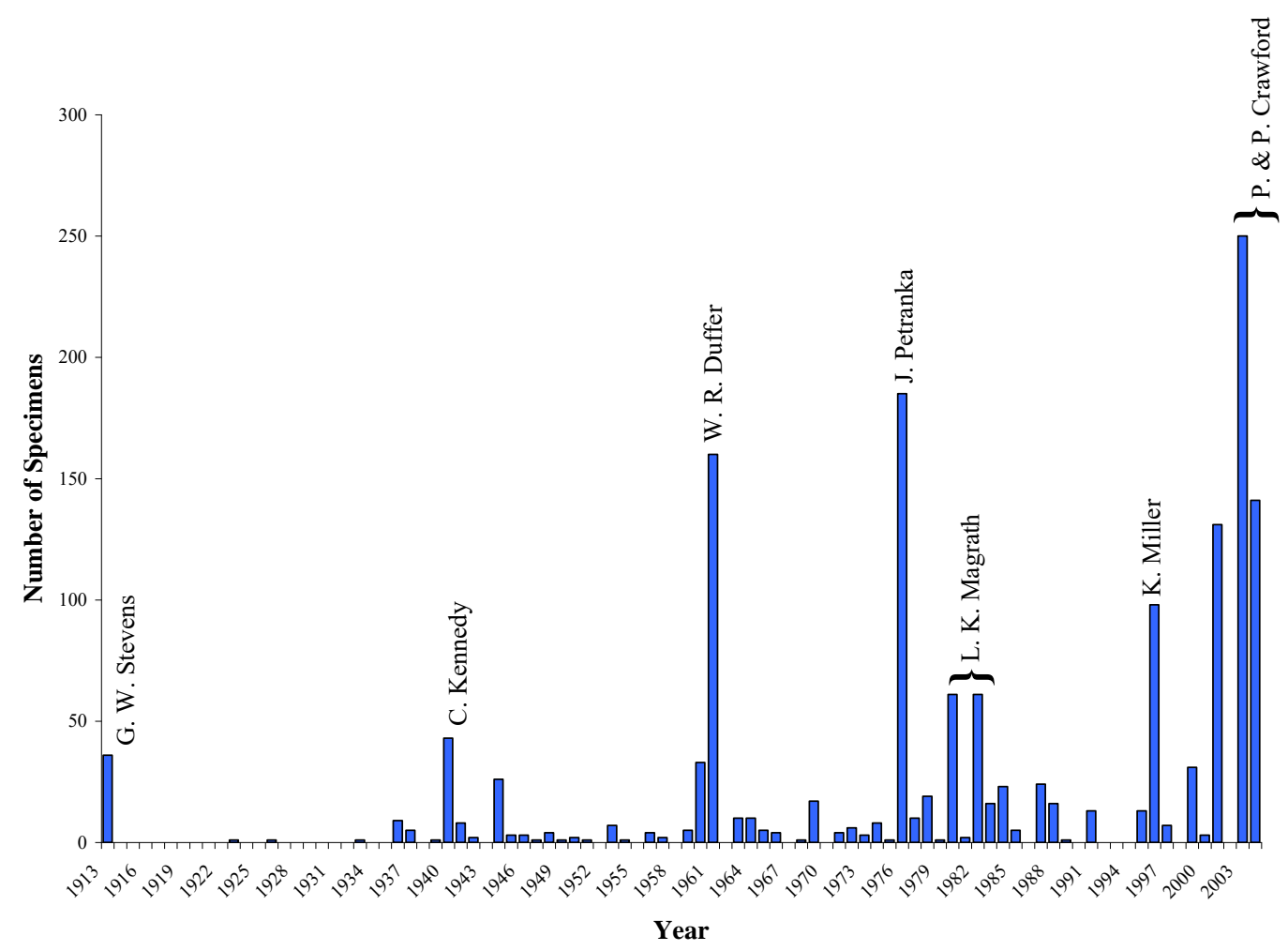

Figure 1 Number of specimens collected per year in Garvin County as represented in the Oklahoma Vascular Plants Database (Hoagland et al. 2004). Collectors responsible for the majority of the collections are listed above the bars. 


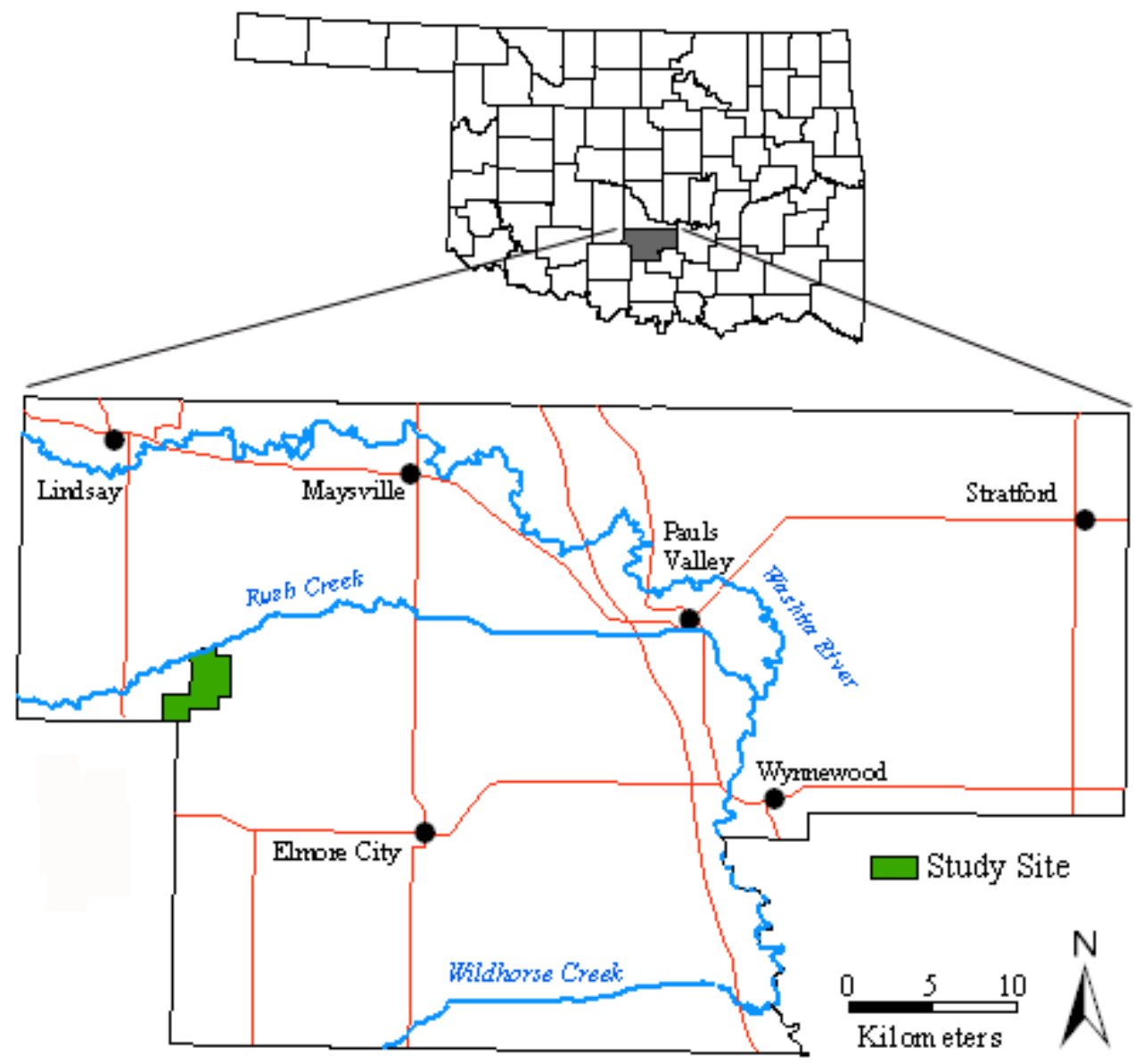

Figure 2 Location of the study site in Garvin County, Oklahoma 


\section{REFERENCES}

Bailey RG. 1995. Description of the ecoregions of the United States. $2 \mathrm{~d}$ ed. rev. and expanded (1 ${ }^{\text {st }}$ ed. 1980). Misc. Publ. No. 1391 (rev.), Washington, DC: USDA Forest Service. 108 p.

Diggs GM, Lipscomb BL, O’Kennon RJ. 1999. Shinners and Mahler's illustrated flora of north central Texas. Fort Worth (TX): Botanical Research Institute of Texas. 1626 p.

Great Plains Flora Association. 1986. Flora of the Great Plains. Lawrence (KS): University Press of Kansas. 1402 p.

Hoagland BW, Buthod AK, Butler IH, Crawford PHC, Udasi AH, Elisens WJ, Tyrl RJ. 2004. Oklahoma Vascular Plants Database. [on-line] Available from http://geo.ou.edu/botanical. Oklahoma Biological Survey, University of Oklahoma, Norman, OK, USA. (accessed 1 October 2004).

Johnson KS, Branson CC, Curtis NM, Ham WE, Harrison WE, Marcher MV, Roberts JF. 1972. Geology and Earth Resources of Oklahoma: an Atlas of Maps and Cross Sections. Oklahoma Geological Survey, Educational Publication I, updated 1979.

Kichler LE, Bogard VA, White JD, Fielder AG, Mobley HL. 1982. Soil survey of Garvin County, Oklahoma. Washington (DC): United States Department of Agriculture. $199 \mathrm{p}$.

Oklahoma Climatological Survey. 2005. Oklahoma Climate Data. [on-line]. Available from http://climate.ocs.ou.edu. University of Oklahoma, Norman, OK, USA. (accessed 6 January 2005).

Oklahoma Natural Heritage Inventory. 2003. ONHI working list of rare Oklahoma plants. [on-line]. Available from http://www.biosurvey.ou.edu/heritage/pu blicat.html. Oklahoma Biological Survey, Norman, OK, USA. (accessed 10 January 2005).

Palmer MW, Wade GL, Neal P. 1995. Standards for the writing of floras. BioScience 45:339345.

Prather LA, Alvarez-Fuentes O, Mayfield MH, Ferguson CJ. 2004. The decline of plant collecting in the United States: a threat to the infrastructure of biodiversity studies. Systematic Botany 29:15-28.

Trewartha GT. 1968. An Introduction to Climate. New York: McGraw-Hill. 399 p. Tyrl RJ, Barber SC, Buck P, Estes JR, Folley P, Magrath LK, Taylor CS, Thompson RA. 2002. Identification of Oklahoma plants: a taxonomic treatment comprising keys and descriptions for the vascular plants of Oklahoma. Stillwater (OK): Flora of Oklahoma Inc. 139 p.

USDA, NRCS. 2004. The PLANTS Database, Version 3.5 [on-line] Available from http://plants.usda.gov. National Plant Data Center, Baton Rouge, LA 70874-4490 USA. (accessed 20 October 2004).

Waterfall UT. 1969. Keys to the flora of Oklahoma. Stillwater (OK): Oklahoma State University. 246 p 


\section{APPENDIX 1. Annotated species list for Garvin County, Oklahoma}

After taxon, the collection number is listed. The herbarium symbol is listed for those species collected in the county by individuals other than Crawford and Crawford and deposited in those herbaria.

* Taxon not previously recorded in the OVPD for Garvin County

${ }^{1}$ BRIT = Herbarium at the Botanical Research Institute of Texas; OCLA = Herbarium at University of Science and Arts of Oklahoma; OKL = Bebb Herbarium, University of Oklahoma; OKLA = Herbarium at Oklahoma State University.

${ }^{2} \mathrm{BF}=$ bottomland forest; $\mathrm{CP}=$ cultivated pasture; $\mathrm{D}=$ disturbed; $\mathrm{NP}=$ native prairie; $\mathrm{R}=$ riparian; $\mathrm{RO}$ $=$ rock outcrop [within the native pasture habitat]; $\mathrm{S}=$ seep; $\mathrm{UF}=$ upland forest; and $\mathrm{WA}=$ wetland/aquatic.

${ }^{3} 5$ = abundant, 1 = rare, see Palmer et al. (1995) for a detailed description of scale; $\mathbf{n} / \mathbf{a}$ indicates the taxon was collected outside of the study site and abundance could not be determined.

${ }^{4} \mathrm{n}=$ native; $\mathrm{i}=$ introduced, $\mathrm{c}=$ cultivated (USDA, NRCS 2004).

${ }^{5}$ see Oklahoma Natural Heritage Inventory (2005) for detailed description of rarity rankings.

Taxon $\quad$ Co\#/Herb ${ }^{1} \quad$ Habitat $^{2}$ Abundance $^{3} \quad$ Origin $^{4}$ Rarity $^{5}$

EQUISETOPSIDA

EQUISETACEAE

Equisetum hyemale L. var. affine (Engelm.)

OKL A.A. Eat.

Equisetum laevigatum A. Braun

1470*

Equisetum X ferrissii Clute (pro.sp.)

OKLA

WA 2

WA 2

n

$\mathrm{n}$

$\mathrm{n}$

$\mathrm{n}$

\section{FILICOPSIDA}

\section{DRYOPTERIDACEAE}

Woodsia obtusa (Spreng.) Torr.

$1102 *$

UF

3

$\mathrm{n}$

MARSILEACEAE

Marsilea vestita Hook. \& Grev.

OKLA

$\mathrm{n}$

G5 S1

OPHIOGLOSSACEAE

Botrychium biternatum (Sav.) Underwood 1552*

Botrychium virginianum (L.) Sw.

Ophioglossum engelmannii Prantl

$1215^{*}$

$1216^{*}$

$\mathrm{BF}$

$\mathrm{BF}$

$\mathrm{BF}$

$1087 *$

NP (RO) 2

$\mathrm{n}$

Pellaea atropurpurea (L.) Link

PINOPSIDA

CUPRESSACEAE

Juniperus virginiana L.

1183

UF

3

$\mathrm{n}$

\section{MAGNOLIOPSIDA}

ACANTHACEAE

Justicia americana (L.) Vahl

Ruellia humilis Nutt.

OKLA

1329

Ruellia strepens L.

1131

ACERACEAE

Acer negundo L.

Acer negundo L. var. texanum Pax

OKL

BF

3

n

$\mathrm{n}$ 
Taxon

AIZOACEAE

Trianthema portulacastrum L.

AMARANTHACEAE

Amaranthus palmeri S. Wats.

Froelichia floridana (Nutt.) Moq.

Gossypianthus lanuginosus (Poir.) Moq.

Iresine rhizomatosa Standl.

ANACARDIACEAE

Rhus aromatica Ait.

Rhus copallinum L.

Rhus glabra L.

Rhus lanceolata (Gray) Britt.

Rhus trilobata Nutt.

Toxicodendron pubescens P. Mill.

Toxicodendron radicans (L.) Kuntze

\section{Co\#/Herb ${ }^{1}$}

OKLA

Habitat $^{2} \quad$ Abundance $^{3}$ Origin $^{4}$ Rarity $^{5}$

1340

OCLA

OKL

1577*

1212

$\mathrm{OKL}$

1293

OKL

OKLA

1496*

1547

$\begin{array}{lll}\text { D } & 4 & \mathrm{n} \\ & & \mathrm{n} \\ & & \mathrm{n} \\ \mathrm{R} & 1 & \mathrm{n}\end{array}$

$\begin{array}{lrl}\text { NP (RO) } & 4 & \text { n } \\ & & \text { n } \\ \text { NP } & 4 & \text { n } \\ & & \text { n } \\ & & \mathrm{n} \\ \text { UF } & 2 & \mathrm{n} \\ \text { D, BF, UF } & 4 & \text { n }\end{array}$

\section{APIACEAE}

Ammoselinum butleri (Engelm. ex S. Wats.)

OKL

OKLA

Ammoselinum popei Torr. \& Gray

Chaerophyllum tainturieri Hook.

Chaerophyllum tainturieri Hook. var. tainturieri 1209

Cicuta maculata L.

Daucus pusillus Michx.

Eryngium leavenworthii Torr. \& Gray

Eryngium yuccifolium Michx.

Lomatium foeniculaceum (Nutt.) Coult. \& Rose

Polytaenia nuttallii DC.

Ptilimnium nuttallii (DC.) Britt.

Sanicula canadensis L.

Spermolepis echinata (Nutt. ex DC.) Heller

Torilis arvensis (Huds.) Link

Torilis japonica (Houtt.) DC.

Zizia aurea (L.) W.D.J. Koch

\section{APOCYNACEAE}

Apocynum cannabinum L.

1245

1440

ARISTOLOCHIACEAE

\section{ASCLEPIADACEAE}

Asclepias amplexicaulis $\mathrm{Sm}$.

OCLA

Asclepias asperula (Dcne.) Woods.

Asclepias asperula (Dcne.) Woods.

ssp. capricornu (Woods.) Woods.

Asclepias stenophylla Gray

Asclepias tuberosa $\mathrm{L}$.

Asclepias tuberosa L. ssp. interior Woods.

Asclepias verticillata $\mathrm{L}$.

Asclepias viridiflora Raf.

Asclepias viridis Walt.

OKLA

1301

OKLA

OCLA

1084

1310

OKLA

OKLA

OKLA

1300*

OKLA

OKLA

1309

OCLA

OKL

OCLA

1259

OKL

1372

1352
G4G5 S1S2

$\begin{array}{lll} & & \mathrm{n} \\ & & \mathrm{n} \\ \mathrm{CP}, \mathrm{UF} & 4 & \mathrm{n} \\ \mathrm{NP} & 2 & \mathrm{n} \\ & & \mathrm{n} \\ & & \mathrm{n} \\ \mathrm{NP}(\mathrm{RO}) & 2 & \mathrm{n} \\ \mathrm{NP} & 2 & \mathrm{n} \\ & & \mathrm{n} \\ & & \mathrm{n} \\ & & \mathrm{n} \\ \mathrm{BF}, \mathrm{UF} & 4 & \mathrm{i} \\ & & \mathrm{i} \\ & & \end{array}$

NP 3

$\mathrm{S}$

2

n

$\begin{array}{lll}\text { BF } & 2\end{array}$

$\begin{array}{lll} & & \mathrm{n} \\ & & \mathrm{n} \\ \mathrm{NP} & 3 & \mathrm{n} \\ & & \mathrm{n} \\ \mathrm{NP} & 3 & \mathrm{n} \\ \mathrm{NP} & 3 & \mathrm{n}\end{array}$


Taxon

Cynanchum laeve (Michx.) Pers.

Co\#/Herb ${ }^{1}$

Matelea decipiens (Alexander) Woods.

OCLA

$\begin{array}{lll}\text { Habitat }^{2} & \text { Abundance }^{3} & \text { Origin }^{4} \text { Rarity }^{5} \\ \text { BF } & 2 & n \\ & & n\end{array}$

\section{ASTERACEAE}

Achillea millefolium $\mathrm{L}$.

1236

Achillea millefolium L. var. occidentalis DC.

OCLA

Ambrosia artemisiifolia L.

1395

Ambrosia psilostachya DC.

1522

Ambrosia trifida L.

Ambrosia trifida L. var. texana Scheele

Amphiachyris dracunculoides (DC.) Nutt.

OKL

1435

$1405^{*}$

$1214^{*}$

Antennaria plantaginifolia (L.) Richards.

OKLA

Arnoglossum plantagineum Raf.

OKL

Artemisia ludoviciana Nutt. ssp. mexicana

(Willd. ex Spreng.) Keck

1466

OCLA

Berlandiera X betonicifolia (Hook.) Small (pro sp.)1163*

Bidens bipinnata L.

Bidens frondosa L.

Brickellia eupatorioides (L.) Shinners

Centaurea americana Nutt.

Chrysopsis pilosa Nutt.

Cirsium altissimum (L.) Hill

$1411 *$

$1535^{*}$

OKL

OKLA

1531

$1509 *$

Cirsium undulatum (Nutt.) Spreng.

1320

Conyza canadensis (L.) Cronq.

OKLA

Conyza canadensis (L.) Cronq. var. canadensis

Conyza ramosissima Cronq.

Coreopsis grandiflora Hogg ex Sweet

1390

$1518^{*}$

OKLA

Coreopsis lanceolata $\mathrm{L}$.

1461

Coreopsis tinctoria Nutt.

OKLA

Dracopis amplexicaulis (Vahl) Cass.

OKLA

Echinacea angustifolia DC.

Echinacea pallida (Nutt.) Nutt.

Eclipta prostrata (L.) L.

Elephantopus carolinianus Raeusch.

1265

OKL

1420 *

1473

1106

Erigeron philadelphicus L.

1238

Erigeron strigosus Muhl. ex Willd.

$1432 *$

Eupatorium serotinum Michx.

OCLA

Evax verna Raf. var. verna

Gaillardia aestivalis (Walt.) H. Rock

1294*

OCLA

Gaillardia pulchella Foug.

$\mathrm{D}, \mathrm{NP} \quad \mathrm{n} / \mathrm{i}$

$\mathrm{n}$

NP $4 \quad n$

NP 5

D $n$

$\begin{array}{lll}\text { D } & \mathrm{n}\end{array}$

NP $5-n$

UF 3

$\mathrm{n}$

Gaillardia suavis (Gray \& Engelm.) Britt. \& Rusby1446*

Gamochaeta purpurea (L.) Cabrera

1318

Grindelia lanceolata Nutt. var. texana (Scheele)

Shinners

Grindelia papposa Nesom \& Suh

OKL

1475

OKL

1428

$1543^{*}$

1558

$1491 *$

OKL

Helianthus petiolaris Nutt.

$1391 *$

NP $\quad 4 \quad n$

BF 3 n

WA 2 n

n

NP 4 n

D 3 n

D 3 n

$\mathrm{n}$

D $4 \quad n$

$\mathrm{D}, \mathrm{CP} \quad 3 \quad \mathrm{n}$

NP 2

$n$

NP 4

$\begin{array}{ll}4 & \text { i }\end{array}$

WA $3 \quad n$

BF 3

R 3 n

$\begin{array}{lll}\mathrm{NP} & 4 & \mathrm{n}\end{array}$

R 3

$\begin{array}{lll}\text { NP } & 4\end{array}$

NP $\quad 4 \quad n$

NP 2 n

UF 3

nan

$\begin{array}{lll}\text { D } & 4 & \mathrm{n} \\ & & \mathrm{n} \\ \mathrm{D} & 4 & \mathrm{n} \\ \mathrm{NP} & 3 & \mathrm{n} \\ \mathrm{NP} & 2 & \mathrm{n} \\ \mathrm{NP} & 2 & \mathrm{n} \\ & & \mathrm{n} \\ \mathrm{D}, \mathrm{NP} & 4 & \mathrm{n}\end{array}$

Crawford \& Crawford 
Taxon

Hieracium longipilum Torr.

Hymenopappus scabiosaeus L'Hér. var. corymbosus (Torr. \& Gray) B.L. Turner

Hymenopappus tenuifolius Pursh

Iva аппиа $\mathrm{L}$.

Iva annua L. var. annua

Lactuca floridana (L.) Gaertn.

Lactuca ludoviciana (Nutt.) Riddell

Lactuca serriola $\mathrm{L}$.

Liatris punctata Hook.

Liatris squarrosa (L.) Michx.

Liatris squarrosa (L.) Michx. var. glabrata

(Rydb.) Gaiser

Marshallia caespitosa Nutt. ex DC.

Oligoneuron rigidum (L.) Small var. rigidum

Packera plattensis (Nutt.) W.A. Weber \& A. Löve OKLA

Packera tampicana (DC.) C. Jeffrey

Palafoxia rosea (Bush) Cory var. macrolepis (Rydb.) B.L. Turner \& Morris

Palafoxia rosea (Bush) Cory var. rosea

Parthenium hysterophorus L.

Pluchea odorata (L.) Cass.

Pseudognaphalium obtusifolium (L.)

Hilliard \& Burtt

Pyrrhopappus carolinianus (Walt.) DC.

Pyrrhopappus grandiflorus (Nutt.) Nutt.

Ratibida columnifera (Nutt.) Woot. \& Standl.

Rudbeckia hirta L.

Rudbeckia hirta L. var. pulcherrima Farw.

Rudbeckia missouriensis Engelm. ex C.L.

Boynt. \& Beadle

Silphium laciniatum L.

Solidago canadensis L. var. scabra Torr. \& Gray

Solidago gigantea Ait.

Solidago ludoviciana (Gray) Small

Solidago nemoralis Ait.

Solidago ulmifolia Muhl. ex Willd.

Sonchus asper (L.) Hill

Symphyotrichum divaricatum (Nutt.) Nesom

Symphyotrichum drummondii (Lindl.) Nesom var. texanum (Burgess) Nesom

Symphyotrichum ericoides (L.) Nesom var. ericoides

Symphyotrichum oblongifolium (Nutt.) Nesom

Symphyotrichum oolentangiense (Riddell) Nesom var. oolentangiense

Symphyotrichum patens (Ait.) Nesom var. patens

Symphyotrichum subulatum (Michx.) Nesom

Taraxacum officinale G.H. Weber ex Wiggers

Tetraneuris linearifolia (Hook.) Greene var. linearifolia

Thelesperma filifolium (Hook.) Gray

Tragopogon dubius Scop.

Co\#/Herb ${ }^{1}$

1271

OKL

1343

$1512 *$

$1392 *$

1486

1154

$1430 *$

OKL

1566

1521

1482

1457

1267

1160

OKL

$1417^{*}$

1398

$1575^{*}$

1503

OKL

1090

1171
OCLA

Habitat $^{2} \quad$ Abundance $^{3}$ Origin $^{4}$ Rarity $^{5}$

OKLA

OKLA

$1545^{*}$

OCLA

OCLA

1515*

OCLA

OKLA

1481*

OKLA

OKLA

$1476^{*}$

1501*

OKLA

OCLA

1387 *

$1400^{*}$

1422*

1378*

$\begin{array}{lll}\text { NP } & 2 & \text { n } \\ \text { D } & 3 & \text { i } \\ \text { WA } & 3 & n\end{array}$

NP 3

NP, CP 3

$\mathrm{NP} \quad 3$

NP 3

NP (RO) $3 \quad n$

$\mathrm{n}$

R 2 i

$\begin{array}{lll}\mathrm{NP} & 4 & \mathrm{n}\end{array}$

NP 3

$\begin{array}{lll}\mathrm{S} & 2 & \mathrm{n}\end{array}$

UF 3

$\mathrm{n}$

$\mathrm{n}$

n

i

n

$\mathrm{n}$

$\mathrm{n}$

$\mathrm{n}$

$\mathrm{n}$

n

$\mathrm{n}$

n

NP 2 n

$\begin{array}{ll} & n \\ n\end{array}$

UF 3

$\begin{array}{lll}\text { UF } & 3 & n\end{array}$

$\begin{array}{lll}\text { UF } & 3 & \mathrm{n}\end{array}$

i

n

$\begin{array}{lll}\text { UF } & 3 & \end{array}$

$\begin{array}{lll}\text { NP } & 4 & n\end{array}$

UF n

R 3 n

$\begin{array}{lll}\text { UF } & 3 & \text { n }\end{array}$

WA 3

$\mathrm{n} / \mathrm{i}$

NP 4 n

NP 4


Taxon

\section{Co\#/Herb ${ }^{1}$}

Verbesina encelioides (Cav.) Benth. \& Hook. f. ex Gray

Verbesina virginica $\mathrm{L}$.

Vernonia baldwinii Torr.

Xanthium strumarium L.

\section{BIGNONIACEAE}

Campsis radicans (L.) Seem. ex Bureau

\section{BORAGINACEAE}

Buglossoides arvensis (L.) I.M. Johnston

Heliotropium tenellum (Nutt.) Torr.

Lithospermum incisum Lehm.

Onosmodium molle Michx. ssp. bejariense

(DC. ex A. DC.) Cochrane

\section{BRASSICACEAE}

Brassica juncea (L.) Czern.

Camelina microcarpa DC.

Capsella bursa-pastoris (L.) Medik.

Cardamine hirsuta L.

Descurainia pinnata (Walt.) Britt.

Draba brachycarpa Nutt. ex Torr. \& Gray

Draba cuneifolia Nutt. ex Torr. \& Gray

Lepidium austrinum Small

Lepidium densiflorum Schrad.

Lepidium virginicum L.

Lesquerella gracilis (Hook.) S. Wats. ssp. nuttallii (Torr. \& Gray) Rollins \& Shaw

Lesquerella ovalifolia Rydb. ex Britt.

Lesquerella ovalifolia Rydb. ex Britt. ssp. alba (Goodman) Rollins \& Shaw

Lesquerella ovalifolia Rydb. ex Britt.

ssp. ovalifolia

Rorippa nasturtium-aquaticum (L.) Hayek

Sibara virginica (L.) Rollins

\section{CACTACEAE}

Echinocereus reichenbachii (Terscheck ex Walp.) Haage f. var. reichenbachii

Escobaria missouriensis (Sweet) D.R. Hunt Escobaria missouriensis (Sweet) D.R. Hunt var. missouriensis

Opuntia macrorhiza Engelm.

\section{CAMPANULACEAE}

Lobelia cardinalis L.

Lobelia siphilitica L.

Triodanis holzingeri McVaugh

\section{CAPRIFOLIACEAE}

Lonicera albiflora Torr. \& Gray

Lonicera flava Sims

Lonicera japonica Thunb.

Sambucus nigra L. ssp. canadensis (L.) R. Bolli
OKLA

1280

1200

OKLA

OKL

$\mathrm{OKL}$

1195

OKL

1217

OKL

1193

OKLA

OCLA

OKLA

OKLA

OCLA

OKL

1189

1204*

OKL

OKL

$1272 *$

1149*

1490

$1555^{*}$

$1418^{*}$

OKLA

R

$\mathrm{S}$

NP (RO) 4

WA 2

$\mathrm{n} / \mathrm{i}$

$\mathrm{n}$

OKLA

1061

OKL

1275

D, WA 4 n

$\begin{array}{lll}\text { UF } & 3 & \mathrm{n} \\ & & \mathrm{n} \\ \mathrm{R} & 3 & \mathrm{i}\end{array}$

Habitat $^{2} \quad$ Abundance $^{3}$ Origin $^{4}$ Rarity $^{5}$

$\begin{array}{lll}\mathrm{R} & 2 & \mathrm{n}\end{array}$

D, NP 4

UF 3

\section{i}

$\mathrm{n}$

$\mathrm{n}$

$\mathrm{n}$

i

i

i

n

$\mathrm{n}$

$\mathrm{n}$

$\mathrm{n}$

$\mathrm{n}$

$\mathrm{n}$

$\mathrm{n}$

$\mathrm{n}$

n

Crawford \& Crawford 
Taxon

Symphoricarpos orbiculatus Moench

Viburnum rufidulum Raf.

CARYOPHYLLACEAE

Arenaria serpyllifolia $\mathrm{L}$.

Cerastium glomeratum Thuill.

Cerastium nutans Raf.

Minuartia michauxii (Fenzl) Farw. var. michauxii

Minuartia michauxii (Fenzl) Farw. var. texana

(B.L. Robins.) Mattf.

Paronychia jamesii Torr. \& Gray

Paronychia virginica Spreng.

Spergularia salina J.\& K. Presl

Stellaria media (L.) Vill.

CELASTRACEAE

Celastrus scandens L.

\section{CHENOPODIACEAE}

Chenopodium album L.

Chenopodium pratericola $\mathrm{Rydb}$.

Chenopodium simplex (Torr.) Raf.

Chenopodium standleyanum Aellen

Cycloloma atriplicifolium (Spreng.) Coult.

Salsola tragus L.

\section{CONVOLVULACEAE}

Convolvulus arvensis L.

Evolvulus nuttallianus J.A. Schultes

Ipomoea hederacea Jacq.

Ipomoea pandurata (L.) G.F.W. Mey.

Ipomoea purpurea (L.) Roth

Ipomoea shumardiana (Torr.) Shinners

CORNACEAE

Cornus drummondii C.A. Mey.

CUCURBITACEAE

Cucurbita foetidissima Kunth

Melothria pendula $\mathrm{L}$.

CUSCUTACEAE

Cuscuta pentagona Engelm.

EBENACEAE

Diospyros virginiana $\mathrm{L}$.

\section{EUPHORBIACEAE}

Acalypha ostryifolia Riddell 1346*

Acalypha virginica $\mathrm{L}$.

Argythamnia mercurialina (Nutt.) Muell.-Arg.

var. mercurialina

Chamaesyce maculata (L.) Small

Chamaesyce missurica (Raf.) Shinners
1353

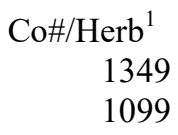

1349

1099

$1210^{*}$

OCLA

OKL

$\mathrm{OKL}$

$1144^{*}$

1407

OCLA

BRIT

OKLA

1289

1464*

$1276^{*}$

$1465^{*}$

$1520^{*}$

OKLA

$1541^{*}$

OCLA

1231

1548

OKLA

$1546^{*}$

OCLA

$1517^{*}$

1258*

$1556^{*}$

$1358^{*}$

1344

$1463 *$

$\begin{array}{lll}\text { Habitat }^{2} & \text { Abundance }^{3} & \text { Origin }^{4} \text { Rarity }^{5} \\ \text { UF } & 5 & \mathrm{n} \\ \text { UF } & 3 & \mathrm{n}\end{array}$

CP, D 3

i

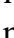

n

NP (RO) $3 \quad n$

NP $4 \quad n$

n

i

BF 2

$\begin{array}{lll}\mathrm{D} & 4 & \mathrm{n} / \mathrm{i}\end{array}$

D 3 n

D 2 n

D $3 \quad n$

$\begin{array}{lll} & & \mathrm{n} \\ \mathrm{D} & 3 & \mathrm{i}\end{array}$

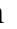

i

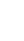

$\mathrm{n}$

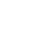

i

n

i

n

D 3 i

n

UF $\quad 4$

D 3 n

UF 2 n

NP $3 \quad n$

UF, R $3 \quad n$

D 3 n

R 2 n

NP 2 n

D 3 n

D 3 n 


\author{
Taxon \\ Chamaesyce nutans (Lag.) Small \\ Chamaesyce prostrata (Ait.) Small \\ Cnidoscolus texanus (Muell.-Arg.) Small \\ Croton capitatus Michx. var. capitatus \\ Croton glandulosus $\mathrm{L}$. \\ Croton glandulosus L. var. lindheimeri \\ Muell.-Arg. \\ Croton glandulosus L. var. septentrionalis \\ Muell.-Arg. \\ Croton monanthogynus Michx. \\ Euphorbia bicolor Engelm. \& Gray \\ Euphorbia commutata Engelm. \\ Euphorbia corollata L. \\ Euphorbia dentata Michx. \\ Euphorbia longicruris Scheele \\ Euphorbia marginata Pursh \\ Euphorbia pubentissima Michx. \\ Euphorbia spathulata Lam. \\ Phyllanthus polygonoides Nutt. ex Spreng. \\ Stillingia sylvatica Garden ex L. \\ Tragia brevispica Engelm. \& Gray \\ Tragia ramosa Torr.
}

\section{FABACEAE}

Acacia angustissima (P. Mill.) Kuntze

Acacia angustissima (P. Mill.) Kuntze var.

hirta (Nutt.) B.L. Robins.

Albizia julibrissin Durazz.

Amorpha canescens Pursh

Amorpha fruticosa $\mathrm{L}$.

Amphicarpaea bracteata (L.) Fern.

Apios americana Medik.

Astragalus crassicarpus Nutt. var. crassicarpus

Astragalus nuttallianus DC.

Astragalus racemosus Pursh

Baptisia australis (L.) R. Br. ex Ait. f. var. minor (Lehm.) Fern.

Baptisia bracteata Muhl. ex Ell. var. leucophaea (Nutt.) Kartesz \& Gandhi

Baptisia sphaerocarpa Nutt.

Cercis canadensis L.

Cercis canadensis L. var. texensis (S. Wats.) M. Hopkins

Chamaecrista fasciculata (Michx.) Greene

Clitoria mariana L.

Dalea aurea Nutt. ex Pursh

Dalea candida Michx. ex Willd.

Dalea candida Michx. ex Willd. var. oligophylla (Torr.) Shinners

Dalea compacta Spreng. var. compacta

Dalea enneandra Nutt.

Dalea purpurea Vent.

Desmanthus illinoensis (Michx.) MacM. ex B.L. Robins. \& Fern.

Desmodium sessilifolium (Torr.) Torr. \& Gray

\begin{tabular}{|c|c|c|c|c|}
\hline Co\#/Herb ${ }^{1}$ & Habitat $^{2}$ & Abundance $^{3}$ & Origin $^{4}$ & Rarity $^{5}$ \\
\hline $1478^{*}$ & $\mathrm{NP}, \mathrm{D}$ & 3 & $\mathrm{n}$ & \\
\hline $1519^{*}$ & $\mathrm{D}$ & 3 & $\mathrm{n}$ & \\
\hline OKLA & & & $\mathrm{n}$ & \\
\hline $1173^{*}$ & NP & 3 & $\mathrm{n}$ & \\
\hline OCLA & & & $\mathrm{n}$ & \\
\hline 1492 & NP & 3 & $\mathrm{n}$ & \\
\hline OKLA & & & $\mathrm{n}$ & \\
\hline 1328 & $\mathrm{D}, \mathrm{NP}$ & 4 & $\mathrm{n}$ & \\
\hline $\mathrm{OKL}$ & & & $\mathrm{n}$ & \\
\hline OCLA & & & $\mathrm{n}$ & G5 S1S2 \\
\hline 1427 & $\mathrm{D}$ & 3 & $\mathrm{n}$ & \\
\hline 1345 & $\mathrm{D}$ & 3 & $\mathrm{n} / \mathrm{i}$ & \\
\hline $1207 *$ & NP (RO) & 4 & $\mathrm{n}$ & \\
\hline 1469 & $\mathrm{NP}, \mathrm{D}$ & 3 & $\mathrm{n}$ & \\
\hline OKLA & & & $\mathrm{n}$ & \\
\hline OKLA & & & $\mathrm{n}$ & \\
\hline OKLA & & & $\mathrm{n}$ & \\
\hline 1229 & NP & 3 & $\mathrm{n}$ & \\
\hline $1148^{*}$ & NP & 4 & $\mathrm{n}$ & \\
\hline OKL & & & $\mathrm{n}$ & \\
\hline OKLA & & & $\mathrm{n}$ & \\
\hline OCLA & & & $\mathrm{n}$ & \\
\hline 1523 & $\mathrm{D}$ & 2 & $\mathrm{i}$ & \\
\hline 1528 & NP & 3 & $\mathrm{n}$ & \\
\hline 1357 & WA & 3 & $\mathrm{n}$ & \\
\hline $1537^{*}$ & UF & 2 & $\mathrm{n}$ & \\
\hline 1576 & $\mathrm{R}$ & 2 & $\mathrm{n}$ & \\
\hline OKL & & & $\mathrm{n}$ & \\
\hline $1091^{*}$ & NP & 4 & $\mathrm{n}$ & \\
\hline OKLA & & & $\mathrm{n}$ & \\
\hline 1445 & NP & 3 & $\mathrm{n}$ & \\
\hline OKLA & & & $\mathrm{n}$ & \\
\hline OKL & & & $\mathrm{n}$ & \\
\hline 1186 & UF & 4 & $\mathrm{n}$ & \\
\hline OKLA & & & $\mathrm{n}$ & \\
\hline 1156 & $\mathrm{D}, \mathrm{NP}$ & 4 & $\mathrm{n}$ & \\
\hline OKL & & & $\mathrm{n}$ & \\
\hline 1264 & NP & 3 & $\mathrm{n}$ & \\
\hline OKLA & & & $\mathrm{n}$ & \\
\hline 1170 & NP & 4 & $\mathrm{n}$ & \\
\hline OKL & & & $\mathrm{n}$ & G5 S1S2 \\
\hline 1370 & NP & 3 & $\mathrm{n}$ & \\
\hline 1263 & NP & 3 & $\mathrm{n}$ & \\
\hline $1333^{*}$ & NP & 4 & $\mathrm{n}$ & \\
\hline 1169 & NP & 3 & $\mathrm{n}$ & \\
\hline
\end{tabular}


Taxon

Desmodium tweedyi Britt.

Gleditsia triacanthos $\mathrm{L}$.

Glottidium vesicarium (Jacq.) Harper

Indigofera miniata Ortega

Indigofera miniata Ortega var. leptosepala

(Nutt. ex Torr. \& Gray) B.L. Turner

Kummerowia stipulacea (Maxim.) Makino

Lathyrus hirsutus L.

Lespedeza procumbens Michx.

Lespedeza stuevei $\mathrm{Nutt}$.

Lespedeza virginica (L.) Britt.

Lotus unifoliolatus (Hook.) Benth.

Medicago lupulina $\mathrm{L}$.

Medicago minima (L.) L.

Medicago sativa $\mathrm{L}$.

Melilotus alba Medikus

Melilotus officinalis (L.) Lam.

Mimosa nuttallii (DC.) B.L. Turner

Neptunia lutea (Leavenworth) Benth.

Oxytropis lambertii Pursh

Oxytropis lambertii Pursh var. articulata

(Greene) Barneby

Pediomelum cuspidatum (Pursh) Rydb.

Pediomelum digitatum (Nutt. ex Torr. \& Gray) Isely

Pediomelum linearifolium (Torr. \& Gray)

J. Grimes

Pediomelum reverchonii (S. Wats.) Rydb.

Prosopis glandulosa Torr.

Psoralidium tenuiflorum (Pursh) Rydb.

Pueraria montana (Lour.) Merr.

Pueraria montana (Lour.) Merr. var. lobata

(Willd.) Maesen \& S. Almeida

Robinia pseudoacacia L.

Sophora affinis Torr. \& Gray

Strophostyles helvula (L.) Ell.

Strophostyles leiosperma (Torr. \& Gray) Piper

Trifolium vesiculosum Savi

Vicia americana Muhl. ex Willd. ssp. minor (Hook.) C.R. Gunn

Vicia sativa $\mathrm{L}$.

\section{FAGACEAE}

Quercus macrocarpa Michx.

Quercus margarettiae Ashe ex Small

Quercus marilandica Muenchh.

Quercus muehlenbergii Engelm.

Quercus prinoides Willd.

Quercus rubra L.

Quercus shumardii Buckl.

Quercus sinuata Walt.

Quercus sinuata Walt. var. breviloba (Torr.)

C.H. Muller

Quercus stellata Wangenh.
Co\#/Herb ${ }^{1}$

$1161^{*}$

1306

1573

1281

1174

$1474 *$

$1138^{*}$

$1530 *$

$1479 *$

1494*

1302

1298*

1456

1542*

OKL

1330

1239

OKLA

1143

OKLA

OKLA

1359 *

OKLA

OKL

OKL

1175

1563

OKLA

1103

OKL

1477*

OKLA

1321

OKLA

1196

1296

1270 *

1202

1286

OKLA

OKL

OKL

OKL

1269

1213
Habitat $^{2} \quad$ Abundance $^{3}$ Origin $^{4}$ Rarity $^{5}$

$\begin{array}{lll}\text { NP } & 3 & n\end{array}$

$\begin{array}{lll}\text { UF } & 4 & n\end{array}$

R, WA 3 n

NP 3 n

NP 3 n

CP, D 2 i

CP 3 i

UF 3 n

NP 3 n

NP 3 n

$\begin{array}{lll}\text { NP } & 2 & n\end{array}$

$\begin{array}{lll}\text { NP } & 3 & \text { i }\end{array}$

CP, D 3 i

D 3 i

D $3-3$

NP 3 n

NP 2 n

NParan

n

n

NP 2 n

n

$\mathrm{n}$

G3 S2

NP $4 \quad n$

D n/a i

UF $-i$

$\begin{array}{lll}\text { UF } & 3 & n\end{array}$

D $4 \quad n$

$\begin{array}{lll}\mathrm{CP} & 3 & \mathrm{n}\end{array}$

CP, D 4 i

BF 3 n

UF 2 n

UF 5 n

UF, BF $4 \quad n$

n

n

n

n G5 S1S2

$\begin{array}{llll}\text { UF } & 2 & \mathrm{n} & \text { G5GT S? }\end{array}$

$\begin{array}{llll}\text { UF } & 5 & \mathrm{n} & \mathrm{G} 5 \mathrm{~S} \text { ? }\end{array}$ 
Taxon

Quercus velutina Lam.
Co\#/Herb ${ }^{1}$

$1185^{*}$
Habitat $^{2}$

UF
Abundance $^{3}$ Origin $^{4}$ Rarity ${ }^{5}$

4

n

$1182 *$

D

3

$\mathrm{n}$

Corydalis curvisiliqua Engelm.

GENTIANACEAE

Centaurium beyrichii (Torr. \& Gray ex Torr.)

B.L. Robins.

1180

Eustoma exaltatum (L.) Salisb. ex G. Don ssp. russellianum (Hook) Kartesz, comb. nov. ined. Sabatia campestris Nutt.

OKLA

1254

GERANIACEAE

Geranium carolinianum L.

Geranium molle L.

$1132 *$

OKL

OKL

$\begin{array}{lll}\text { NP } & 2 & \mathrm{n} \\ & & \mathrm{n} \\ \mathrm{NP} & 3 & \mathrm{n} \\ & & \\ \mathrm{CP}, \mathrm{D} & 4 & \mathrm{n} \\ & & \mathrm{i}\end{array}$

HALORAGACEAE

Myriophyllum heterophyllum Michx.

OKLA

Aesculus glabra Willd. var. arguta (Buck1.)

B.L. Robins.
$1101 *$

1348

1334

1150

Krameria lanceolata Torr.

\section{LAMIACEAE}

Hedeoma drummondii Benth.

Hedeoma hispida Pursh

Lamium amplexicaule $\mathrm{L}$.

Lycopus americanus Muhl. ex W. Bart.

Monarda citriodora Cerv. ex Lag.

Monarda clinopodioides Gray

Monarda fistulosa L.

Monarda fistulosa L. ssp. fistulosa var. mollis

(L.) Benth.

Monarda pectinata Nutt.

Physostegia virginiana (L.) Benth.

Salvia azurea Michx. ex Lam.

Salvia azurea Michx. ex Lam. var. grandiflora Benth.

Salvia reflexa Hornem.

Scutellaria ovata Hill ssp. bracteata (Benth.) Epling

Teucrium canadense L. var. canadense
1172

1242

1085

OKL

OKLA

1248

1256

OKL

OKLA

OKLA

OKL

$$
1397
$$

$1376^{*}$

OKL

1159

OKLA

$\begin{array}{lll}\text { UF } & 2 & \end{array}$

$\begin{array}{lll}\text { BF } & 4 & \mathrm{n} \\ \text { BF } & 3 & n\end{array}$

$\begin{array}{lll}\text { NP } & 3\end{array}$

$\begin{array}{lll}\text { NP } & 3 & n\end{array}$

NP 3 n

CP, D 4 i

$n$

$\mathrm{n}$

$\begin{array}{lll}\text { NP } & 3 & n \\ & 3 & n\end{array}$

\section{LAURACEAE}

Sassafras albidum (Nutt.) Nees n

$\mathrm{n}$

n

n

n

n

$\mathrm{n}$

n

n

n

n

n

n

n 
Taxon

LINACEAE

Linum alatum (Small) Winkl.

Linum berlandieri Hook. var. berlandieri

Linum pratense (J.B.S. Norton) Small

Linum rigidum Pursh

Linum sulcatum Riddell

LOASACEAE

Mentzelia oligosperma Nutt. ex Sims

\section{Co\#/Herb ${ }^{1}$}

Lythrum californicum Torr. \& Gray

\section{MALVACEAE}

Callirhoe alcaeoides (Michx.) Gray

Callirhoe involucrata (Torr. \& Gray) Gray

Sida spinosa L.

\section{MENISPERMACEAE}

Cocculus carolinus (L.) DC.

Menispermum canadense $\mathrm{L}$.

\section{MOLLUGINACEAE}

Mollugo verticillata $\mathrm{L}$.

\section{MORACEAE}

Maclura pomifera (Raf.) Schneid.

Morus alba L.

Morus rubra L.

\section{NELUMBONACEAE}

Nelumbo lutea Willd.

NYCTAGINACEAE

Mirabilis albida (Walt.) Heimerl

Mirabilis linearis (Pursh) Heimerl

\section{OLEACEAE}

Forestiera pubescens Nutt. var. pubescens

Fraxinus americana $\mathrm{L}$.

Fraxinus pennsylvanica Marsh.

Fraxinus texensis (Gray) Sarg.

\section{ONAGRACEAE}

Calylophus berlandieri Spach

Calylophus berlandieri Spach ssp. berlandieri

Calylophus serrulatus (Nutt.) Raven

Gaura mollis James

Ludwigia peploides (Kunth) Raven
$1145 *$

$1240 *$

OKLA

OKLA

OKLA

OKL

1421

OKLA

OKL

OKLA

1369*

1246

$1544 *$

1307

OKLA

1140

OKL

1368

OCLA

$1425 *$

WA, D 3

n

$1550 *$

1385*

OKLA

OCLA

1292

1221

OKL

1153*

1241

OKLA

1341*

Habitat $^{2} \quad$ Abundance $^{3}$ Origin $^{4}$ Rarity $^{5}$

$\begin{array}{lll}\text { NP } & 2 & \mathrm{n} \\ \mathrm{NP} & 4 & \mathrm{n} \\ & & \mathrm{n} \\ & & \mathrm{n} \\ & & \mathrm{n}\end{array}$

$n$

n

n

n

n

n

n

n

n

n

n

UF 4

UF 2 i

UF 3

WA n/a n

D, UF 3

n

OKL

n

n

n

-

n

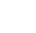


Taxon

Ludwigia peploides (Kunth) Raven ssp. glabrescens (Kuntze) Raven

Oenothera laciniata Hill

Oenothera macrocarpa Nutt.

Oenothera macrocarpa Nutt. ssp. oklahomensis (J.B.S. Norton) Wagner

Oenothera speciosa Nutt.

Stenosiphon linifolius (Nutt. ex James) Heynh.

OXALIDACEAE

Oxalis corniculata $\mathrm{L}$.

Oxalis stricta L.

Oxalis violacea $\mathrm{L}$.

PAPAVERACEAE

Argemone polyanthemos (Fedde) G.B. Ownbey

Passiflora incarnata L.

Passiflora lutea L.

PEDALIACEAE

Proboscidea louisianica (P. Mill.) Thellung

PHYTOLACCACEAE

Phytolacca americana L.

\section{PLANTAGINACEAE}

Plantago patagonica Jacq.

Plantago rhodosperma Dcne.

Plantago virginica $\mathrm{L}$.

\section{PLATANACEAE}

Platanus occidentalis L.

\section{POLEMONIACEAE}

Ipomopsis rubra (L.) Wherry

Phlox pilosa L.

\section{POLYGALACEAE}

Polygala alba Nutt.

\section{POLYGONACEAE}

Eriogonum alatum Torr.

Eriogonum alatum Torr. var. glabriusculum

Torr.

Eriogonum annuum Nutt.

Eriogonum longifolium Nutt.

Polygonum aviculare L.

Polygonum hydropiperoides Michx.

Polygonum lapathifolium L.

Polygonum pensylvanicum L.

Polygonum punctatum Ell.

Polygonum ramosissimum Michx.
1223

$1431^{*}$

$1565^{*}$

Co\#/Herb ${ }^{1}$

OKLA

1255

$1443 *$

1224

OCLA

1404

1582

OKLA

1092

$1436^{*}$

1342

$1233^{*}$

OKL

1208*

OKLA

OKL

1139

1093

1406

OCLA

$1559 *$

$1402 *$

1386

1471

1308

1412

OKLA

1525*
Habitat $^{2} \quad$ Abundance $^{3}$ Origin $^{4}$ Rarity $^{5}$

$n$

$\begin{array}{lll}\mathrm{D} & 3 & \mathrm{n}\end{array}$

D, NP $3 \quad n$

D, NP (RO) $3 \quad n$

D, NP $4 \quad n$

CP, D, NP $3 \quad n$

NP 3

D $4 \quad n$

UF, BF 2 n

UF, BF 2 n

D 3 n

UF, D 3

NP 3

NP 3

n

n

NP 2 n

NP (RO) 4

NP (RO) $3 \quad n$

G5 S2S3

$n$

$\begin{array}{lll}\mathrm{D} & 3 & \mathrm{i}\end{array}$

WA 3 n

WA 3 n

WA 3 n

WA 2 n

Crawford \& Crawford 
Taxon

Polygonum scandens L. var. cristatum

(Engelm. \& Gray) Gleason

Polygonum virginianum $\mathrm{L}$.

Rumex altissimus Wood

Rumex crispus $\mathrm{L}$.

Rumex hastatulus Baldw.

PORTUlaCACEAE

Claytonia virginica $\mathrm{L}$.

\section{$\mathrm{Co \# /Herb}{ }^{1}$}

$1557 *$

OKLA

1327

OKLA
OKLA

Habitat $^{2} \quad$ Abundance $^{3}$ Origin $^{4}$ Rarity $^{5}$

$\begin{array}{lll}\text { R } & 2 & \mathrm{n} \\ & & \mathrm{n} \\ \text { D, WA } & 3 & \mathrm{n} \\ & & \mathrm{i} \\ & & \mathrm{n}\end{array}$

OKL

$\mathrm{n}$

1086

Dodecatheon meadia L.

Samolus valerandi L. ssp. parviflorus (Raf.) Hultén 1506*

\section{RANUNCULACEAE}

Anemone berlandieri Pritz.

1188

Clematis pitcheri Torr. \& Gray

Delphinium carolinianum Walt.

Ranunculus hispidus Michx.

Ranunculus sceleratus $\mathrm{L}$.

Thalictrum dasycarpum Fisch. \& Avé-Lall.

Thalictrum revolutum DC.

OKLA

1244

OKL

1569*

1578

OKLA

$\begin{array}{lll}\text { NP } & 2 & \mathrm{n}\end{array}$

WA 3 n

RHAMNACEAE

Ceanothus americanus $\mathrm{L}$.

Ceanothus herbaceus Raf.

1444

OKL

$\begin{array}{lll}\text { NP } & 3 & \mathrm{n} \\ & & \mathrm{n} \\ \text { NP } & 3 & \mathrm{n} \\ & & \mathrm{n} \\ \text { WA } & 2 & \mathrm{n} \\ \text { BF } & 2 & \mathrm{n} \\ & & \mathrm{n}\end{array}$

\section{ROSACEAE}

Crataegus crus-galli $\mathrm{L}$.

Crataegus mollis Scheele

OCLA

OKL

OKLA

Crataegus viridis $\mathrm{L}$.

Geum canadense Jacq.

Geum canadense Jacq. var. texanum

Fern. \& Weatherby

Prunus angustifolia Marsh.

Prunus gracilis Engelm. \& Gray

Prunus mexicana S. Wats.

Rosa foliolosa Nutt. ex Torr. \& Gray

Rosa multiflora Thunb. ex Murr.

Rubus oklahomus Bailey

1363

OKLA

OKL

1203

1371

OKL

1097

OKLA

NP (RO) 2

n

n

1303

1493

1218

$1502 *$

1279

1199

Hedyotis nigricans (Lam.) Fosberg

Houstonia pusilla Schoepf

\section{RUTACEAE}

Ptelea trifoliata $\mathrm{L}$.

Zanthoxylum americanum P. Mill.
OKL

OKL

$\mathrm{n}$
$\mathrm{n}$
$\mathrm{n}$
$\mathrm{i}$

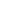

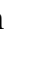

$$
\text { n }
$$

n

n

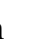

Diodia teres Walt.

Galium aparine L.

$\begin{array}{lll}\text { WA } & 3 & \mathrm{n} \\ \text { NP } & 3 & \mathrm{n} \\ \text { CP, UF } & 4 & \mathrm{n} \\ \text { UF } & 3 & \mathrm{n} \\ \text { NP } & 4 & \mathrm{n} \\ \text { NP } & 4 & \mathrm{n}\end{array}$

Crawford \& Crawford 
Taxon

SALICACEAE

Populus deltoides Bartr. ex Marsh.

Salix caroliniana Michx.

Salix exigua Nutt.

Salix nigra Marsh.

\section{Co\#/Herb ${ }^{1}$}

$1228 *$

OKL

1572

1105

\section{SAPINDACEAE}

Sapindus saponaria L. var. drummondii

(Hook. \& Arn.) L. Benson

SAPOTACEAE

Sideroxylon lanuginosum Michx. ssp. oblongifolium (Nutt.) T.D. Pennington

\section{SCROPHULARIACEAE}

Agalinis heterophylla (Nutt.) Small ex Britt.

Bacopa rotundifolia (Michx.) Wettst.

Buchnera americana L.

Castilleja indivisa Engelm.

Castilleja purpurea (Nutt.) G. Don var. citrina (Pennell) Shinners

Castilleja sessiliflora Pursh

Leucospora multifida (Michx.) Nutt.

Nuttallanthus texanus (Scheele) D.A. Sutton

Penstemon cobaea Nutt.

Penstemon oklahomensis Pennell

Scrophularia marilandica L.

Verbascum thapsus L.

Veronica anagallis-aquatica L.

Veronica peregrina L. ssp. xalapensis (Kunth) Pennell

\section{SOLANACEAE}

Physalis angulata L.

Physalis cinerascens (Dunal) A.S. Hitchc.

Physalis longifolia Nutt. var. longifolia

Physalis mollis Nutt. var. mollis

Physalis pubescens L. var. pubescens

Physalis pumila Nutt.

Solanum americanum P. Mill.

Solanum carolinense L.

Solanum dimidiatum Raf.

Solanum elaeagnifolium Cav.

Solanum ptychanthum Dunal

Solanum rostratum Dunal

\section{TAMARICACEAE}

Tamarix chinensis Lour.

Tamarix gallica L.

\section{ULMACEAE}

Celtis laevigata Willd.

Celtis laevigata Willd. var. reticulata (Torr.) L. Benson

Celtis laevigata Willd. var. texana Sarg.
1331

$1257^{*}$

1505

OCLA

1278

1151

OKL

OKL

1499*

30

1152

OKLA

OKLA

OKLA

1570 *

$1108^{*}$

OKL

1311

1347*

OKLA

1434*

OKL

OKLA

OKLA

1319

1268

OKL

1350

1220*

OKLA

Habitat $^{2} \quad$ Abundance $^{3}$ Origin $^{4}$ Rarity $^{5}$

$\begin{array}{lll}\text { R, S } & 3 & \mathrm{n} \\ & & \mathrm{n} \\ \text { R, WA } & 3 & \mathrm{n} \\ \text { R } & 3 & \mathrm{n}\end{array}$

UF

4

n

$\mathrm{BF}, \mathrm{UF} \quad 3$

n

$\begin{array}{lll}\text { NP } & 3 & \mathrm{n} \\ & & \mathrm{n} \\ \text { NP } & 2 & \mathrm{n} \\ \text { NP } & 4 & \mathrm{n}\end{array}$

$\mathrm{n}$

D, WA 3 n

D, NP 3 n

NP 3

G3 S3

WA 2

D 2

n

$\begin{array}{lll}\mathrm{n} & \\ \mathrm{n} & & \\ \mathrm{n} & & \\ \mathrm{n} & & \\ \mathrm{n} & \\ \mathrm{n} & \\ \mathrm{n} & \\ \mathrm{n} & \end{array}$

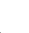

n

n


Taxon

Celtis tenuifolia Nutt.

Co\#/Herb ${ }^{1}$

OKL

1184

$1201 *$

Ulmus rubra Muhl.

URTICACEAE

Boehmeria cylindrica (L.) Sw.

OKLA

OKLA

OKLA

$\begin{array}{lll}\text { Habitat }^{2} & \text { Abundance }^{3} & \text { Origin }^{4} \text { Rarity }^{5} \\ & & \mathrm{n} \\ \text { BF, UF } & 5 & \mathrm{n} \\ \mathrm{BF} & 3 & \mathrm{n}\end{array}$

n

n

n G5 S2

$1107 *$

S, R 3

n

1351

OKL

1190

$1510 *$

OKLA

1458*

$1455^{*}$

$1579 *$

1168

1274

1198

OKLA

$\begin{array}{lll}\text { NP } & 3 & \mathrm{n} \\ & & \mathrm{n} \\ \text { NP } & 4 & \mathrm{n} \\ \text { WA } & 2 & \mathrm{n} \\ & & \mathrm{n} \\ \text { NP } & 3 & \mathrm{n} \\ \text { D } & 3 & \mathrm{n} \\ \text { R } & 2 & \mathrm{n} \\ \text { NP } & 3 & \mathrm{n}\end{array}$

\section{VIOLACEAE}

Viola affinis Le Conte

BF, UF $4 \quad n$

Viola bicolor Pursh

Viola sororia Willd.

\section{VISCACEAE}

Phoradendron leucarpum (Raf.) Reveal \&

M.C. Johnston

OKL

Phoradendron tomentosum (DC.) Engelm. ex Gray 1305*

\section{VITACEAE}

Cissus incisa auct. non Des Moulins

Cissus trifoliata (L.) L.

OKL

1364

Parthenocissus quinquefolia (L.) Planch.

$1222 *$

Vitis cinerea (Engelm.) Millard

$1366^{*}$

Vitis cinerea (Engelm.) Millard var. cinerea

Vitis vulpina L.

CP, D 3

n

$\mathrm{n}$

BF, UF $3 \quad n$

\section{ZYGOPHYLLACEAE}

Tribulus terrestris L.

1468

1147

OKL

OCLA

OKL

OKLA

NP

3

$\mathrm{n}$

$\mathrm{NP}(\mathrm{RO}), \mathrm{R} 2 \mathrm{n}$

BF, UF 4 n

BF, UF 4 n

BF, UF 4 n

BF, UF 4 n

D 3 i

Crawford \& Crawford 
Taxon

Co\#/Herb ${ }^{1}$

Sagittaria platyphylla (Engelm.) J.G. Sm. $\begin{array}{lll}\text { Habitat }^{2} & \text { Abundance }^{3} & \text { Origin }^{4} \text { Rarity }^{5} \\ \text { WA } & n / a & n\end{array}$

$\mathrm{D}$

4

$\mathrm{n}$

OKLA

OCLA

OCLA

$\mathrm{n}$

$\mathrm{n}$

$\mathrm{n}$
OKL

OCLA

OKLA

OCLA

OKLA

OCLA

OKLA

OKL

OKLA

Carex muehlenbergii Schkuhr ex Willd.

Carex muehlenbergii Schkuhr ex Willd. var. enervis Boott

OCLA

OCLA

OKLA

Carex tetrastachya Scheele

1356

1529

1488

1325

$1526^{*}$

$1178^{*}$

1581

OCLA

OKLA

1205*

OKLA

OKLA

is compressa Sullivant

1514

Eleocharis palustris (L.) Roemer \& J.A. Schultes

Fimbristylis annua (All.) Roemer \& J.A. Schultes

Fimbristylis puberula (Michx.) Vahl

OCLA

OKL

Fimbristylis puberula (Michx.) Vahl var. puberula

1232

Fimbristylis vahlii (Lam.) Link

1571

Fuirena simplex Vahl

Fuirena simplex Vahl var. aristulata (Torr.) Kral 1487

Fuirena simplex Vahl var. simplex

1498

Fuirena squarrosa Michx.

OCLA

OCLA

1235

Rhynchospora nivea Boeckl.

Schoenoplectus americanus (Pers.) Volk. ex

Schinz \& R. Keller

Schoenoplectus pungens (Vahl) Palla

OKLA

$1285^{*}$

Schoenoplectus tabernaemontani (K.C. Gmel.)

Palla

OKLA

OKLA

$1322 *$
Scirpus lineatus Michx.

Scirpus pendulus Muhl.

$\mathrm{n}$
$\mathrm{n}$
$\mathrm{n}$
$\mathrm{n}$

n

n

n

n

n

n

n

n

n

n

n

WA 3 n

S 3 n

D $3 \quad n / i$

S 3 n

D 3 n

S 3 n

WA 1

$\mathrm{n}$

S 3 n

$\mathrm{n}$

WA, S 3 n

n

n

$\begin{array}{lll}\text { NP } & 4 & \end{array}$

WA 2 n

$\begin{array}{lll}\mathrm{S} & 3 & \mathrm{n}\end{array}$

$\begin{array}{lll}\mathrm{S} & 3 & \mathrm{n}\end{array}$

S 3 n

$n$

S 4

G4 S2

Crawford \& Crawford 
Taxon

IRIDACEAE

Hypoxis hirsuta (L.) Coville

Nemastylis geminiflora Nutt.

Nemastylis nuttallii Pickering ex R.C. Foster

Sisyrinchium angustifolium P. Mill.

Sisyrinchium campestre Bickn.

\section{JUNCACEAE}

Juncus bufonius L.

Juncus dudleyi Wieg.

Juncus interior Wieg.

Juncus marginatus Rostk.

Juncus nodatus Coville

Juncus scirpoides Lam.

Juncus tenuis Willd.

Juncus torreyi Coville

\section{LEMNACEAE}

Lemna aequinoctialis Welw.

\section{LILIACEAE}

Allium canadense L.

Allium canadense L. var. fraseri Ownbey

Allium drummondii Regel

Allium stellatum Nutt. ex Ker-Gawl.

Androstephium caeruleum (Scheele) Greene

Nothoscordum bivalve (L.) Britt.

Polygonatum biflorum (Walt.) Ell.

NAJADACEAE

Najas guadalupensis (Spreng.) Magnus

\section{Co\#/Herb ${ }^{1}$}

OKL

OKL

OCLA

1243

OKLA

OCLA

$1460 *$

$1284^{*}$

1176

OKLA

OKLA

$1374 *$

1472

$1549 *$

OCLA

1095

$1096 *$

OCLA

1191

1192

$1129 *$

OCLA
Habitat $^{2} \quad$ Abundance $^{3}$ Origin $^{4}$ Rarity $^{5}$

1564* NP 2 n

OCLA

1410

OCLA

$1135^{*}$

OKL

$1452 *$

$1459 *$

1426

OKL

POACEAE

Agrostis hyemalis (Walt.) B.S.P.

Agrostis perennans (Walt.) Tuckerman

Alopecurus carolinianus Walt.

Andropogon gerardii Vitman

Andropogon hallii Hack.

Andropogon glomeratus (Walt.) B. S. P. var.

hirsutior (Hack.) C. Mohr

$1415 *$

1401

$1266^{*}$

Aristida oligantha Michx.
Aristida purpurea Nutt. var. purpurea

$1394 *$

Bothriochloa ischaemum (L.) Keng var. songarica
(Rupr. ex Fisch. \& C.A. Mey.) Celarier \& Harlan

Bothriochloa laguroides (DC.) Herter ssp.

torreyana (Steud.) Allred \& Gould

$1297 *$

Bothriochloa saccharoides (Sw.) Rydb.

$\begin{array}{ccc} & & \mathrm{n} \\ & & \mathrm{n} \\ \mathrm{NP} & 4 & \mathrm{n} \\ & & \mathrm{n} \\ & & \mathrm{n}\end{array}$

$\begin{array}{lll} & & \mathrm{n} \\ \text { WA } & 2 & \mathrm{n} \\ \text { WA } & 2 & \mathrm{n} \\ \text { S } & 2 & \mathrm{n} \\ & & \mathrm{n} \\ & & \mathrm{n} \\ \text { BF } & 3 & \mathrm{n} \\ \text { WA, S } & 3 & \mathrm{n}\end{array}$

WA $\quad 3$

$\begin{array}{lll} & & \mathrm{n} \\ \mathrm{NP} & 3 & \mathrm{n} \\ \mathrm{NP} & 3 & \mathrm{n} \\ & & \mathrm{n} \\ \mathrm{NP} & 4 & \mathrm{n} \\ \mathrm{NP} & 4 & \mathrm{n} \\ \mathrm{BF} & 2 & \mathrm{n}\end{array}$

NP 3

$\mathrm{n}$

$\mathrm{n}$

$\mathrm{n}$

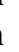

n

$n$

$n$
$n$
$n$
$n$

n

D 4 i

$\begin{array}{lll}N P & 3 & n\end{array}$

WA 3

NP 4 n

n

S, R 3 n

$\begin{array}{lll}\text { NP } & 4 & n\end{array}$

NP $4 \quad n$

$\begin{array}{lll}\text { NP } & 3 & \text { i }\end{array}$

NP 4

n 


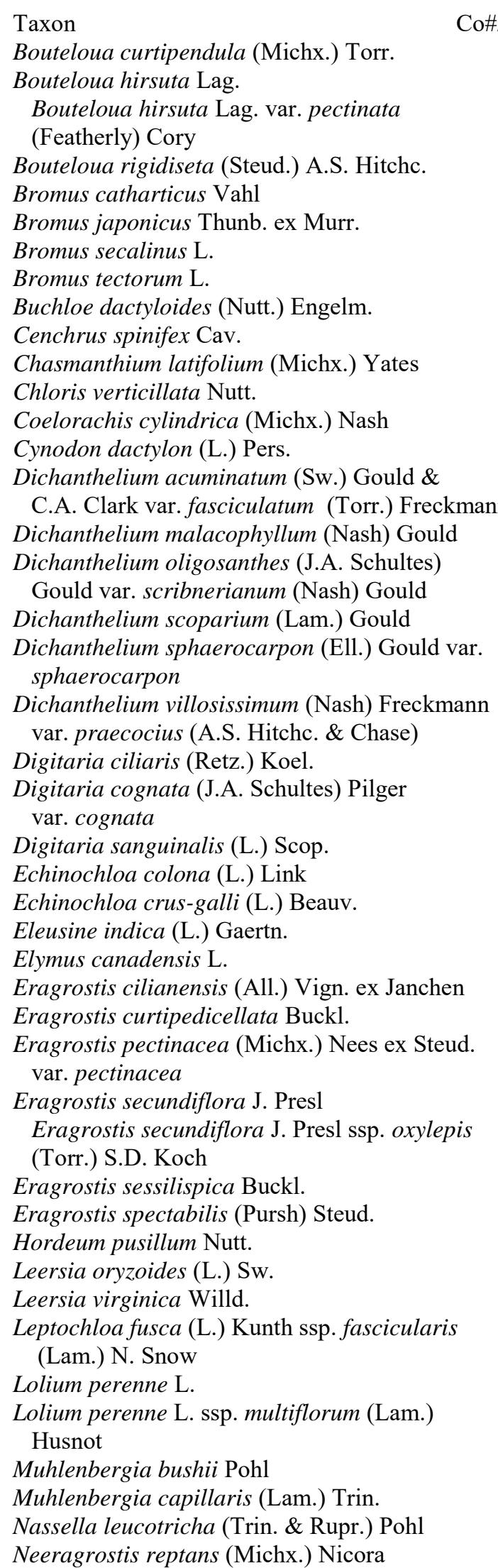

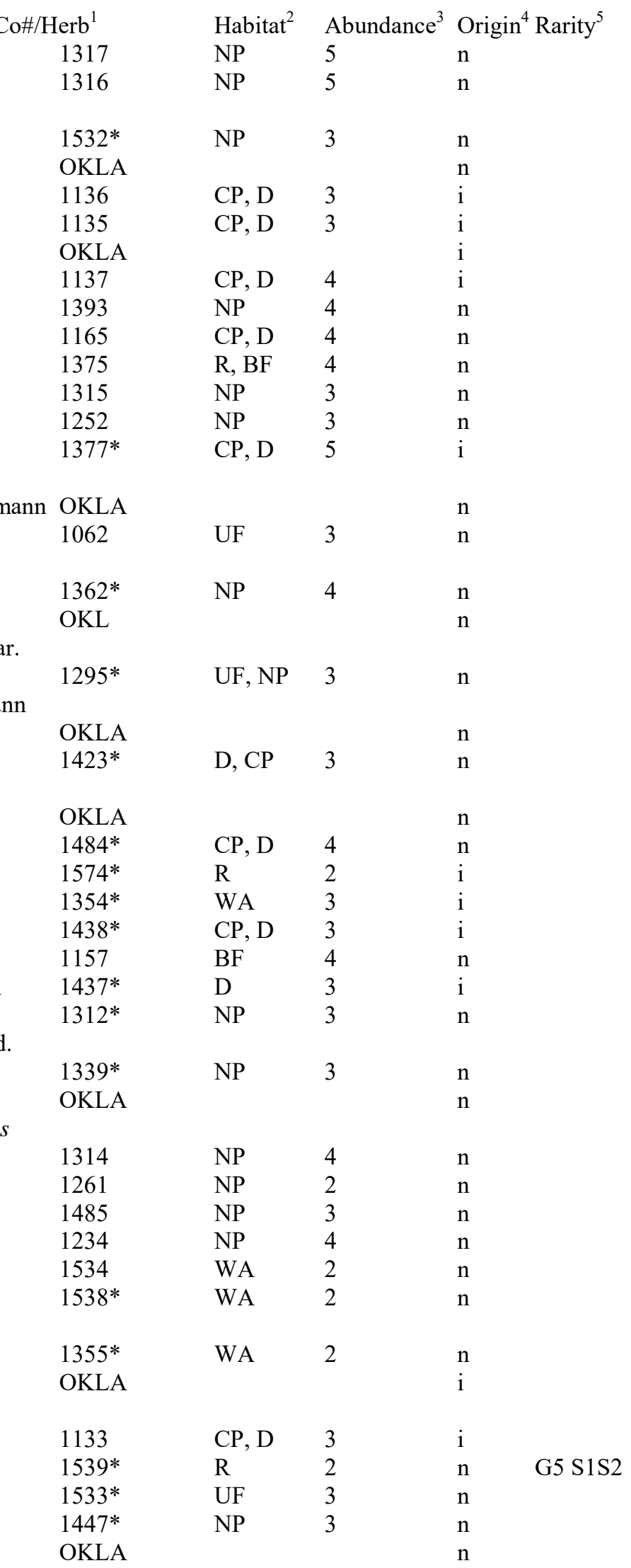


Taxon

Panicum acuminatum Sw. var. lindheimeri (Nash) Lelong

Panicum anceps Michx.

Panicum capillare L.

Panicum dichotomiflorum Michx.

Panicum obtusum Kunth

Panicum philadelphicum Bernh. ex Trin.

Panicum virgatum $\mathrm{L}$.

Pascopyrum smithii (Rydb.) A. Löve

Paspalum distichum $\mathrm{L}$.

Paspalum floridanum Michx.

Paspalum laeve Michx.

Paspalum notatum Flueggé

Paspalum pubiflorum Rupr. ex Fourn.

Paspalum setaceum Michx.

Poa аппиа L.

Poa arachnifera Torr.

Poa bulbosa L.

Poa pratensis L.

Polypogon monspeliensis (L.) Desf.

Schizachyrium scoparium (Michx.) Nash

Sclerochloa dura (L.) Beauv.

Secale cereale L.

Setaria parviflora (Poir.) Kerguélen

Setaria pumila (Poir.) Roemer \& J.A. Schultes

Setaria viridis (L.) Beauv.

Sorghastrum nutans (L.) Nash

Sorghum halepense (L.) Pers.

Sporobolus clandestinus (Biehler) A.S. Hitchc.

Sporobolus compositus (Poir.) Merr.

Sporobolus cryptandrus (Torr.) Gray

Tridens flavus (L.) A.S. Hitchc.

Triticum aestivum $\mathrm{L}$.

Urochloa texana (Buckl.) R. Webster

PONTEDERIACEAE

Heteranthera limosa (Sw.) Willd.

\section{Co\#/Herb ${ }^{1}$}

1553*

1414

1483*

1527

1373

1433*

1462

1164*

OKL

OKLA

1424*

OKLA

1313

1500

1449 *

1451

OKL

OKL

OKLA

1403*

1067*

1454*

OKLA

1158*

1337

1396

1166

$1416^{*}$

$1409^{*}$

1338

1408 *

$1453 *$

1540 *

OCLA

1507 *

1098

OKL

1554*
Habitat $^{2} \quad$ Abundance $^{3}$ Origin $^{4}$ Rarity $^{5}$

$\begin{array}{lll}\mathrm{R} & 2 & \mathrm{n}\end{array}$

S, WA 3 n

NP 4 n

NP 3 n

NP 3 n

BF 2 n

S, R $3 \quad n$

NP $4 \quad n$

n

R, D $3 \quad n$

R, D $3 \quad n$

D 3 n

D 4 i

BF 3 n

n/i

NP 5

NP 2

D 2

CP, D $3 \quad$ i

CP, D $4 \quad$ i

NP 5 n

D $\quad 4 \quad$ i

NP 3 n

NP $3 \quad n$

NP $3 \quad n$

NP $4 \quad n$

D 3 i

R 2 n

POTAMOGETONACEAE

Potamogeton nodosus Poir.

\section{SMILACACEAE}

Smilax bona-nox L.

Smilax rotundifolia $\mathrm{L}$.

Smilax tamnoides L.

OKL

WA 2 n

TYPHACEAE

Typha angustifolia $\mathrm{L}$.

$\begin{array}{lll}\text { BF, UF } & 4 & \mathrm{n} \\ \text { BF, UF } & 3 & \mathrm{n} \\ & & \end{array}$

ZANNICHELLIACEAE

Zannichellia palustris L.

OCLA

n 